\title{
Stiffness Analysis of Co-axle Sleeve Coupling with Fan and Laminations
}

\author{
Xuguang Min $^{\mathrm{a}}$, Jun Fan ${ }^{\mathrm{b}}$, Ying Jiang ${ }^{\mathrm{c}}$ \\ Jiangxi Science \& Technology Normal University, Nanchang, China \\ aminxuguang@vip.163.com, bfangjun020j13@163.com, cpljy0791@163.com
}

Keywords: diesel generator set; coupling; laminations; stiffness; fan.

Abstract. We propose a novel compact coupling for diesel generator sets, in which the coupling laminations and the generator fans share one bushing, thereby reducing the overall axial length of the generator set. The maximum stress and elastic deformation during operation were determined by finite element analysis to determine the optimum stiffness of the laminations, and the error of the analysis was discussed, providing a reference for the structural improvement and future design of couplings for diesel generator sets.

\section{Introduction}

Currently, metal laminated membrane couplings are preferably used as couplings between diesel engines and generators because they allow to compensate the axis offset between the major and the minor axes, show a high torsion stiffness, high-speed adaptability, no need for lubrication or maintenance, a good adaptability to various working conditions, and they also exhibit a good vibration and noise reduction performance compared with rigid couplings and flexible couplings with rubber components. They are also increasingly used to replace gear couplings.

However, so far, in domestically and internationally available commercial diesel generator sets, the metal lamination couplings and the generator fans are two separate parts, with both parts requiring bushings either as the body of the fan blades or the coupling laminations. This structure not only increases the axial length, weight and volume of the coupling, but also makes it more difficult to process and assemble the parts. Combining the coupling and the generator fan of diesel generators into a single unit would not only allow to reduce the axial dimension of the unit by at least one sleeve length but also to reduce the number of parts and assembling procedures, thereby lowering the production costs and shortening the manufacturing cycle of the unit.

\section{Coupling design}

Concerning the assembly of the diesel generator components, by directly installing the coupling laminations on the fan shaft sleeve, the proposed design saves the need to install a separate sleeve on the coupling laminations of the generator, thus shortening the axial dimension of the generator rotor shaft and reducing the total weight.

The number of screw holes on the fan sleeves increased to directly fix the coupling laminations on the fan hub. These changes will not affect the performance of the fan or the coupling. because the diesel generator mainly cools the generator through radial ventilation; air is sucked in from the surroundings at the rear end of the generator, flowing towards the generator front along the axial direction, and then turning $90^{\circ}$ to form a radial outflow through the fan. The functioning of couplings laminations relies on their own elastic deformation. As long as there is a distance of $5 \mathrm{~mm}$ or sufficient clearance between the lamination and the fan ribs or blades, interference will not occur.

Of course, the clearance between the coupling lamination and the fan ribs or blades should ensure that the fan ribs or blades do not interfere with any parts of the diesel engines. A front view and side view of the proposed coupling are shown in Fig. 1. The fan blade (1) is welded on the sleeve (2), which is connected to the generator shaft (3) via a flat key (4); the reinforcement (5) is welded on the outer end of the blade (1); the lamination (9) is pressed against the end face of the sleeve (2) by a screw (8); the baffle (6) is pressed against the end face of the lamination (9) by another screw (7). 

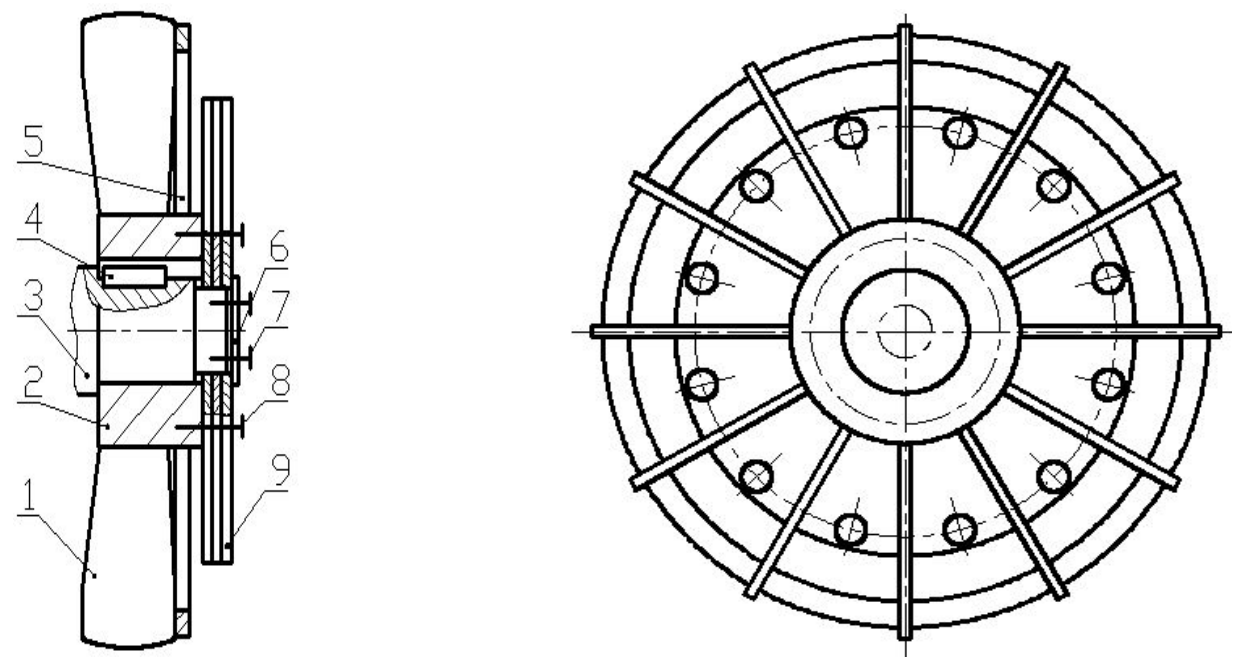

Fig.1 View of the proposed coupling

\section{Stiffness analysis of coupling-lamination}

The design of diesel generator couplings aims not only at the transmission of the rated torque but also at compensating for the misalignment error, which is related to finding the optimum lamination stiffness. Practice has shown that, if the stiffness is too high, the performance of the coupling lamination stack will be similar to the performance of traditional rigid couplings, and thus cannot compensate for the misalignment error, resulting in excessive deformation and damage to the generator's other low-stiffness parts. On the other hand, if the stiffness is too low, the deformation of the coupling laminations will be too high during operation, leading to collision accidents between the outer peripheral surface of the rotor core and the bore surface of the stator core.

Therefore, for this study, we assumed that the stiffness is at its optimum when the elastic deformation of the coupling lamination stack compensates for the misalignment error.

According to the law of conservation of energy conservation and the Lagrange dynamic equations, the general equation describing the rotor dynamics in matrix form can be expressed as:

$$
M \ddot{y}(t)+C \dot{y}(t)+K y(t)=F(t)
$$

where $\mathrm{M}, \mathrm{C}$ and $\mathrm{K}$ are the mass matrix, the damping matrix, and the stiffness matrix of the coupling, respectively, $\mathrm{t}$ is time, $\mathrm{y}$ is a generalized coordinate, and $\mathrm{F}$ is the force vector. $\mathrm{M}, \mathrm{C}$ and $\mathrm{K}$ are constant matrices for a particular diesel generator set. When designing the structure of a lamination stack, the structure is usually designed first, followed by a verification of its stiffness to check if it is qualified and optimal. Modern finite element analysis (FEA) software has been developed to automate the calculation process employing classical mechanics to determine the stiffness of machine parts. Users just need to know the structure, material, dimensions, loading and other boundary conditions, and can then obtain the corresponding mechanical properties. The lamination stack consists of four laminations, and a schematic illustration of the coupling lamination design is shown in Fig.2.The international system of units was used, and the heat resistant 0Cr18Ni10Ti austenitic stainless steel was selected as lamination material, with a slice thickness of $0.002 \mathrm{~m}$ each, an allowable yield strength $\sigma_{\mathrm{s}}$ of $160 \mathrm{MPa}$, poisson's ratio $v$ of 0.3 and an elastic modulus E of 210GPa.The SOLID186 structural solid model was selected as the unit type for the analysis of the lamination stack. The global grid cell size was selected to $0.08 \mathrm{~m}$ for division, and mapping division was used. Under load, the 12 holes on the outside of the laminations all restrict the freedom of movement, with a displacement of $0.0001 \mathrm{~m}$ in all three directions (Ux, Uy and $\mathrm{Uz}$ ), which is the preset misalignment displacement of the compensation unit. During operation, the laminations are fixed on the flywheel of the diesel engine through the 12 holes on the outside, and then fixed on the coupling of the generator through the 12 holes on the inside. The torque (here: 3,200 $\mathrm{Nm}$ ) is transmitted through friction between the laminations. The laminations bore should be able to 
bear the pressure of $7.5 \mathrm{MPa}$ caused by the partial centrifugal force of the generator's rotor, as well as the unilateral magnetic force, etc. The load diagram for the laminations is shown in Fig.3.

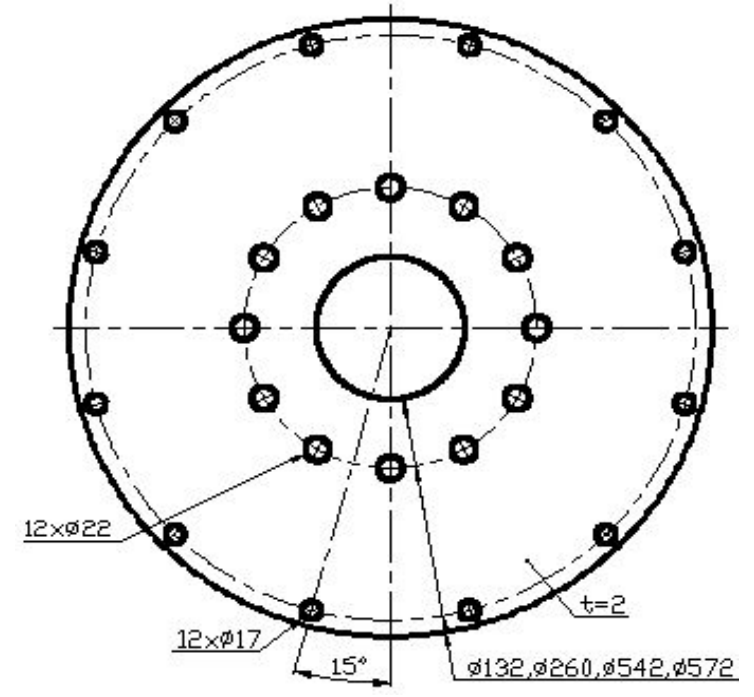

Fig.2 A diagram of lamination

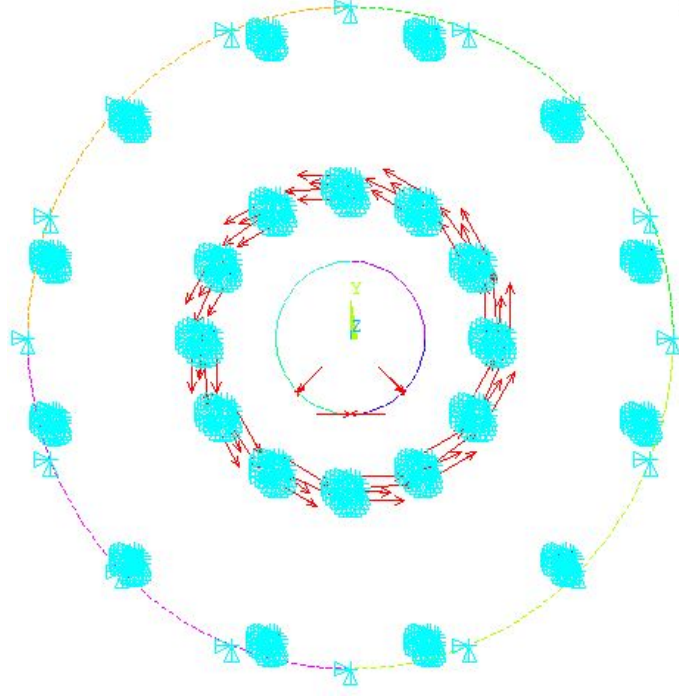

Fig.3 A diagram of laminations loading

The maximum stress occurring in the laminations is shown in Fig.4; the maximum elastic deformation occurring in the laminations is revealed in Fig.5; and the error range of the performed FEA is shown in Fig.6. The maximum stress in the laminations is $132 \mathrm{MPa}$, as shown in Figure 5, which is lower then the allowed yield strength $(160 \mathrm{MPa})$ of the lamination material. The maximum elastic deformation occurring in the laminations is $0.2 \mathrm{~mm}$, as shown in Fig.5, suggesting that the proposed stiffness design is reasonable and meets the project requirements. Fig. 6 shows that the error of the FEA is below $6.6 \%$, which is lower than $12 \%$, and is therefore within a range acceptable in general engineering.

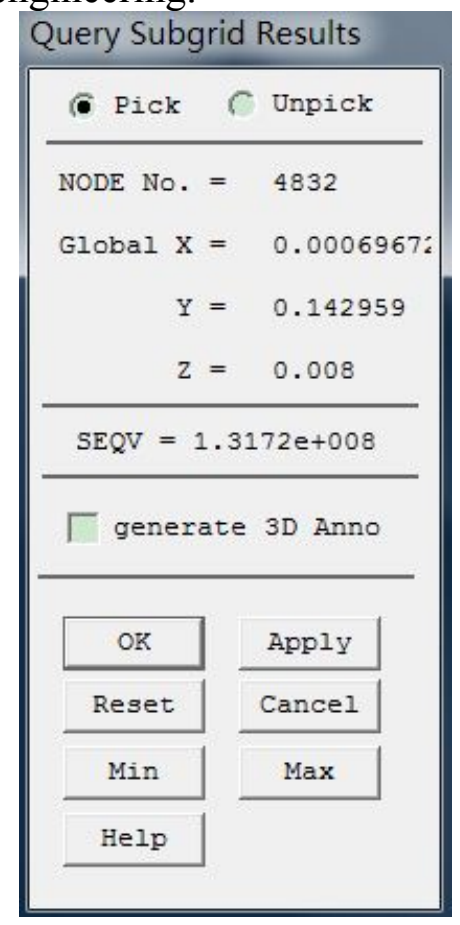

Fig.4 Lamination's maximum stress

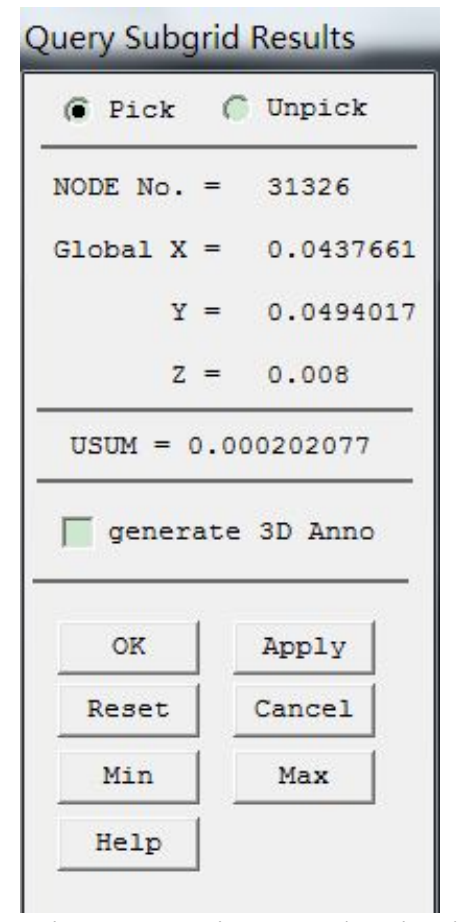

Fig.5 Lamination's maximum elastic deformation

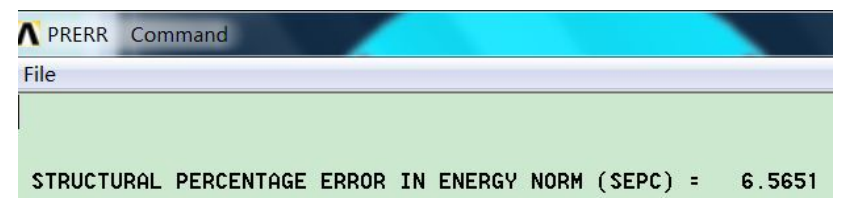

Fig.6 Error range for the finite element analysis of the laminations 


\section{Summary}

In this paper, we reported on a novel compact coupling for a diesel generator set. The coupling laminations and the generator fans share a sleeve, which shortens the axial length of the whole generator set. The maximum stress and elastic deformation during operation were determined by finite element analysis, and the error of the analysis was discussed, which provides a reference for the structural improvement and future design of couplings for diesel generator sets.

\section{Acknowledgements}

This study was supported by the Science \& Technology Plan Program of Jiangxi Province, China (Grant No. 20132BAB206027).

\section{References}

[1] Liu ZL, Yao HL, Li LX, Wen BC. Lateral stiffness calculation method of diaphragm couplings . Chinese Journal of Construction Machinery, 2013,11(2):103-106.

[2] Min XG,Li GZ,Wang Q,Wang GQ.Design for the quantity of coupling disk in diesel generator set. Machinery Design \& Manufacture.2009,7:45-47.

[3] Min XG, Wan T, Zeng XL. Laminated membrane coupling with the sleeve of fan: China, 2013205054598 [P].2014-07-23.

[4] Deng GL, Zhao YB, Song D, Xing LF. Contact state of the laminated membrane subassemblies of strength and stiffness impact analysis. Ship Science and Technology,2010,32(8):157-161.

[5] Shentu LF, Sun CL. Calculation of laminated stiffness of joint flexible laminated membrane coupling[J]. Journal of Mechanical Strength, 2013,35(4):531-536.

[6] Min XG,Xiong ZK.Application of matlab in design of generator rotor shaft.S \& M Electric Machines,2004,31(3):55-57.

[7] W.Wang.The Strength-Fatigue Life and Dynamic Characteristics Analysis of a Type of Laminated Membrane Coupling. Nanjing: Nanjing University of Aeronautics and Astronautics,2013.

[8] H.Liao.Finite element analysis and structure optimum design rese-arch for diaphragm of diaphragm coupler.Chong Qing:Chongq-ing University,2008.

[9] M.Cheng.Research on Laminated Membrane Coupling Based on Nonlinear Finite Element Method. Nanjing: Nanjing University of Aeronautics and Astronautics, 2006.

[10]B.Zhou,L.Lin.Compound stress analysis of the flexible metal-disc couplings. Journal of Mechanical Transmission. 2009,33(4):93-97. 